\title{
Optimal control-based methodology for active vibration control of pedestrian structures
}

\author{
Emiliano Pereira ${ }^{\mathrm{a}, *}$, Iván M. Díaz ${ }^{\mathrm{b}}$, Emma J. Hudson ${ }^{\mathrm{c}}$, Paul Reynolds $^{\mathrm{c}, \mathrm{d}}$ \\ ${ }^{a}$ Escuela Politécnica Superior, Universidad de Alcalá, ES 28805, Alcalá de Henares, \\ Madrid, Spain \\ ${ }^{b}$ ETS Ingenieros de Caminos, Universidad Politécnica de Madrid, ES 28040, Madrid, \\ Spain \\ ${ }^{c}$ College of Engineering, Mathematics and Physical Sciences, University of Exeter, \\ EX4 4QF, Exeter, UK \\ ${ }^{d}$ Full Scale Dynamics Limited, 40 Leavygreave Road, S3 $7 R D$, Sheffield, UK
}

\begin{abstract}
Civil structures such as floor systems with open-plan layouts or lightweight footbridges can be susceptible to excessive levels of vibrations caused by human activities. Active vibration control (AVC) via inertial-mass actuators has been shown to be a viable technique to mitigate vibrations, allowing structures to satisfy vibration serviceability limits. It is generally considered that the determination of the optimal placement of sensors and actuators together with the output feedback gains leads to a tradeoff between the regulation performance and the control effort. However, the "optimal" settings may not have the desired effect when implemented because simplifications assumed in the control scheme components may not be valid and/or the actuator/sensor limitations are not considered. This work proposes a design methodology for multi-input multi-output vibration control of pedestrian
\end{abstract}

\footnotetext{
*Corresponding author: Tel.: +34 918856711; Fax: +34 926295361; Email: Emiliano.Pereira@uah.es
} 
structures to simultaneously obtain the sensor/actuator placement and the control law. This novel methodology consists of minimising a performance index that includes all the significant practical issues involved when inertialmass actuators and accelerometers are used to implement a direct velocity feedback in practice. Experimental results obtained on an in-service indoor walkway confirm the viability of the proposed methodology.

Keywords: Active vibration control, Inertial-mass actuators, MIMO control, Human-induced vibrations, Floor vibrations, Optimal control.

\section{Introduction}

Improvements in design and construction methods have led to light and slender floor structures which have increased susceptibility to vibration. This is exacerbated by the current trend towards design of more open-plan structures. Examples of significant vibrations due to human-induced excitations have been found in open-plan floors and footbridges, as well as other structures $[1,2]$. These structures satisfy ultimate limit state criteria but have the potential of attracting complaints due to excessive human-induced vibrations [3]. Active vibration control (AVC) via inertial-mass actuators has been shown to significantly reduce the level of response, allowing otherwise excessively lively structures to satisfy vibration serviceability limits. However, AVC is a relatively new area of research in the civil engineering community and, as such, there are a number of obstacles that must be overcome before the field can mature fully [4]. One of these obstacles is the limitations of inertial-mass actuators, such as force and stroke saturations and low-frequency response. Single-input single-output (SISO) control strategies 
dealing with these problems have been proposed $[5,6,7]$. Here, the stability of the overall system is guaranteed and the sensitivity to stroke saturation, which can damage the inertial-mass actuator hardware, is alleviated.

It has been shown that the use of only one inertial-mass actuator may limit the number of controlled vibration modes since the mode shape of a mode to be controlled should have sufficiently large amplitude at the control location. In addition, the dynamics of inertial-mass actuators also limit the maximum damping imparted to a structure. One obvious solution is to use multiple SISO control schemes, which are designed independently for each location (this strategy is commonly denoted as multi-SISO control). Although multi-SISO control can be a viable solution [8], it may be of limited efficiency since the structural system does not act independently at each control location (i.e., a force applied at one location will influence the structural response at another location for every mode shape that is non-zero at both locations).

A better performance can be achieved if a multi-input multi-output (MIMO) control strategy is used. This was shown in [9], where an optimal direct output velocity feedback (DVF) MIMO controller was presented. This DVF MIMO control strategy finds the optimal gain matrix and the optimal location for a predefined number of actuators and sensors. The optimal sensor/actuator placement and the gain matrix is obtained by minimising a performance index (PI) that considers the amplitude and duration of the vibration and the maximum force imparted for each actuator. Simulation results were presented in [9], demonstrating the advantages of using MIMO control as opposed to SISO control. However, the controller proposed in [9] considers an ideal DVF limited only by the maximum actuator force. To im- 
plement DVF using inertial-mass actuators, the following additional issues have to be carefully considered:

- the actuator bandwidth (i.e., frequency response) significantly affects the stability of the overall control scheme and limits the maximum damping imparted to the structure,

- the actuator stroke saturation, which also limits the maximum damping imparted, could result in dramatic adverse effects on the actuator performance and its hardware,

- the velocity is obtained by integrating the output signal of an accelerometer, necessitating the use of a lossy integrator, which affects the stability of the control scheme,

- a low-pass filter may be required to guarantee the finite gain property of the control loop at high frequencies, avoiding spillover problems [10], and

- the frequency bandwidth where humans perceive the vibration [11] may be considered to focus the control effort on the most important vibration modes.

These issues were not considered in [9] and hence the method presented there is not implementable as such. The work presented here builds on the idea presented in [9] and considers the aforementioned practical issues to propose a novel control design methodology. This methodology is illustrated by designing and testing an AVC for an in-service indoor walkway. 
This paper is organised as follows. Section 2 explains the control scheme elements paying special attention to the inclusion of the practical issues into the closed-loop and to the definition of a weighted state vector that takes into account the human vibration perception. Section 3 details the design methodology. Section 4 provides the description of the in-service indoor walkway and the experimental implementation of the design methodology on the structure. Section 5 concludes the paper.

\section{Control scheme}

This section explains the general scheme shown in Fig. 1 used to define an optimal DVF MIMO control from the proposed optimisation design process, which is also included in this section. The dynamics included in Fig. 1 are grouped into the following blocks:

1. The flexible structure, such as a floor or lightweight footbridge, which is modelled by $n$ vibration modes. The inputs are the force generated by $p$ actuators $\left(\mathbf{u}_{s}\right)$ and $r$ perturbations $\left(\mathbf{w}_{s}\right)$. The accelerations measured by a set of accelerometers at $q$ different locations $\left(\mathbf{y}_{a}\right)$ are considered as control outputs.

2. The additional dynamics needed to obtain the velocity from the accelerometers are denoted as lossy integrators. The lossy integrators are considered as ideal integrators plus high-pass filters [12]. Thus, each lossy integrator carries out the magnitude and phase shift of an ideal integrator at frequencies above the cut-off frequency of the high-pass filter whilst removing any DC component and avoiding unnecessary high sensitivity to stroke saturation at low frequencies. 
3. The control gain matrix and the required low-pass filters, which are required to guarantee the finite gain property of the control loop at high frequencies, avoiding spillover problems [10].

4. The saturation nonlinearity models the actuator force limitation, which is limited by the maximum power amplifier input. This maximum value can be decreased to reduce the risk of stroke saturation but also reducing the actuator performance.

5. The dynamics of the inertial-mass actuators.

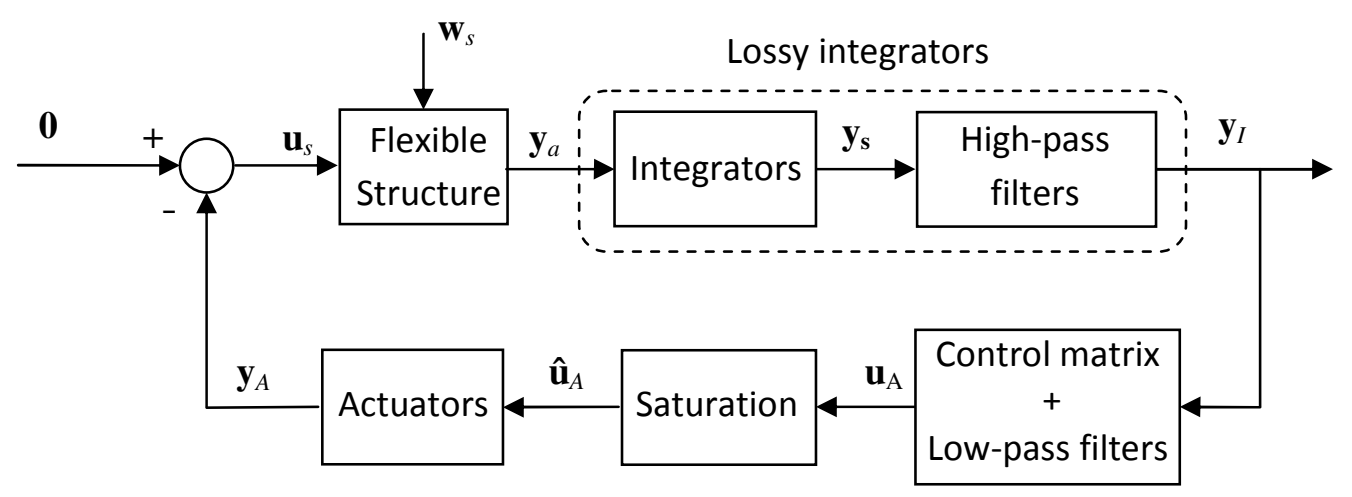

Figure 1: General control scheme.

\subsection{Description of the control scheme components}

For the sake of simplicity, the flexible structure and the integrators are grouped so that the output of the resulting system is $\mathbf{y}_{s}$, which is the velocity at $q$ locations. Thus, the standard state-space representation of the model for this flexible structure is represented as follows:

$$
\begin{array}{r}
\dot{\mathbf{x}}_{s}=\mathbf{A}_{s} \mathbf{x}_{s}+\mathbf{B}_{s_{1}} \mathbf{u}_{s}+\mathbf{B}_{s_{2}} \mathbf{w}_{s} \\
\mathbf{y}_{s}=\mathbf{C}_{s} \mathbf{x}_{s} .
\end{array}
$$


If model (1) is defined in modal coordinates, the state-space matrices are as follows [13]:

$$
\begin{gathered}
\mathbf{A}_{s}=\left[\begin{array}{cc}
\mathbf{0} & \mathbf{I} \\
-\Omega^{2} & -2 \mathbf{Z} \Omega
\end{array}\right], \quad \mathbf{B}_{s_{1}}=\left[\begin{array}{c}
\mathbf{0} \\
\boldsymbol{\Phi}_{\boldsymbol{u}}
\end{array}\right], \\
\mathbf{B}_{s_{2}}=\left[\begin{array}{c}
\mathbf{0} \\
\boldsymbol{\Phi}_{\boldsymbol{w}}
\end{array}\right],
\end{gathered}
$$

where $\Omega$ is a $n \times n$ diagonal matrix formed by the natural frequencies $\left(\left[\omega_{1}, \cdots, \omega_{n}\right]\right), \mathbf{Z}$ is a $n \times n$ diagonal matrix formed by the damping ratios $\left(\left[\zeta_{1}, \cdots, \zeta_{n}\right]\right)$ and $\boldsymbol{\Phi}_{\boldsymbol{u}}, \boldsymbol{\Phi}_{\boldsymbol{y}}$ and $\boldsymbol{\Phi}_{\boldsymbol{w}}$ are matrices with dimensions $n \times p, q \times n$ and $n \times r$, respectively. Each $k^{\text {th }}$ column of $\boldsymbol{\Phi}_{\boldsymbol{u}}$ and $\boldsymbol{\Phi}_{\boldsymbol{w}}$ and each row of $\boldsymbol{\Phi}_{\boldsymbol{y}}$ is formed by the $k^{t h}$ vibration mode values at the positions of the actuators $\left(\boldsymbol{\Phi}_{\boldsymbol{u}}\right)$, perturbations $\left(\boldsymbol{\Phi}_{\boldsymbol{w}}\right)$ and sensors $\left(\boldsymbol{\Phi}_{\boldsymbol{y}}\right)$.

The high-pass filters utilised in this work are second-order Butterworth high-pass filters with cut-off frequency equal to $\omega_{I}$. The chosen value of $\omega_{I}$ is the result of the tradeoff between the resonance frequency of actuator, since small values of $\omega_{I}$ increase the risk of stroke saturation, and the first vibration mode of the structure, since higher values of $\omega_{I}$ reduce the damping imparted by a DVF controller. The state-space model of each high-pass filter is as follows:

$$
\begin{array}{r}
\dot{\mathbf{x}}_{I}=\mathbf{A}_{I_{T}} \mathbf{x}_{I}+\mathbf{B}_{I_{T}} \mathbf{y}_{s} \\
\mathbf{y}_{I}=\mathbf{C}_{I_{T}} \mathbf{x}_{I}+\mathbf{D}_{I_{T}} \mathbf{y}_{s}
\end{array}
$$

being the matrices $\mathbf{A}_{I_{T}}=\operatorname{diag}\left(\mathbf{A}_{I}, \cdots, \mathbf{A}_{I}\right), \mathbf{B}_{I_{T}}=\operatorname{diag}\left(\mathbf{B}_{I}, \cdots, \mathbf{B}_{I}\right)$, $\mathbf{C}_{I_{T}}=\operatorname{diag}\left(\mathbf{C}_{I}, \cdots, \mathbf{C}_{I}\right)$ and $\mathbf{D}_{I_{T}}=\operatorname{diag}\left(\omega_{I}^{4}, \cdots, \omega_{I}^{4}\right)$ block diagonal, where 
$\mathbf{A}_{I}, \mathbf{B}_{I}$ and $\mathbf{C}_{I}$ are defined as follows [14]:

$$
\mathbf{A}_{I}=\left[\begin{array}{cc}
0 & 1 \\
-\omega_{I}^{2} & -2 \sqrt{2} \omega_{I}
\end{array}\right], \mathbf{B}_{I}=\left[\begin{array}{l}
0 \\
1
\end{array}\right], \mathbf{C}_{I}=\left[\begin{array}{cc}
-\omega_{I}^{4} & -2 \sqrt{2} \omega_{I}^{3}
\end{array}\right]
$$

The control gain matrix $(\mathbf{K})$ in a general form is defined as:

$$
\boldsymbol{K}=\left[\begin{array}{cccc}
K_{11} & K_{12} & \cdots & K_{1 q} \\
K_{21} & K_{22} & \cdots & K_{2 q} \\
\vdots & \vdots & \ddots & \vdots \\
K_{p 1} & K_{p 2} & \cdots & K_{p q}
\end{array}\right]
$$

in which $K_{p q}$ is the control gain applied at control input $p$ due to control output $q$.

The low-pass filters to avoid spillover problems [10] are defined as follows:

$$
\begin{array}{r}
\dot{\mathbf{x}}_{L P}=\mathbf{A}_{L P_{T}} \mathbf{x}_{L P}+\mathbf{B}_{L P_{T}} \mathbf{y}_{I} \\
\mathbf{y}_{L P}=\mathbf{C}_{L P_{T}} \mathbf{x}_{L P},
\end{array}
$$

being the matrices $\mathbf{A}_{L P_{T}}=\operatorname{diag}\left(\mathbf{A}_{L P}, \cdots, \mathbf{A}_{L P}\right), \mathbf{B}_{L P_{T}}=\operatorname{diag}\left(\mathbf{B}_{L P}, \cdots, \mathbf{B}_{L P}\right)$ and $\mathbf{C}_{L P_{T}}=\operatorname{diag}\left(\mathbf{C}_{L P}, \cdots, \mathbf{C}_{L P}\right)$ block diagonal, where $\mathbf{A}_{L P}, \mathbf{B}_{L P}$ and $\mathbf{C}_{L P}$ are defined as follows [14]:

$$
\mathbf{A}_{L P}=\left[\begin{array}{cc}
0 & 1 \\
-\omega_{L P}^{2} & -2 \sqrt{2} \omega_{L P}
\end{array}\right], \mathbf{B}_{L P}=\left[\begin{array}{l}
0 \\
1
\end{array}\right], \mathbf{C}_{L P}=\left[\begin{array}{ll}
\omega_{L P}^{2} & 0
\end{array}\right],
$$

The value of $\omega_{L P}$, which is the cut-off frequency, must be sufficiently high when compared with the controlled vibration mode with the maximum resonance frequency.

The outputs of the saturation block, which are the command voltage inputs of the $p$ actuators, are denoted by $\hat{\mathbf{u}}_{A}$. The actuator considered is 
an inertial actuator that generates forces through acceleration of an inertial mass to the structure on which it is placed. The actuator consists of an inertial (or moving) mass $m_{A}$ attached to a current-carrying coil moving in a magnetic field created by an array of permanent magnets. The inertial mass is connected to the frame by a suspension system. The mechanical part is modelled by a spring stiffness $k_{A}$ and a viscous damping $c_{A}$. The electrical part is modelled by the resistance $R$, the inductance of the coil $L$ and the voice coil constant $C_{E}$, which relates coil velocity and the back electromotive force (Fig. 2(a)) [15]. Combining the mechanical and the electrical part, the linear behaviour of the actuator can be closely described as a third-order dynamic model. Thus, the state space model of the $p$ actuators is as follows:

$$
\begin{array}{r}
\dot{\mathbf{x}}_{A}=\mathbf{A}_{A_{T}} \mathbf{x}_{A}+\mathbf{B}_{A_{T}} \hat{\mathbf{u}}_{A} \\
\mathbf{y}_{A}=\mathbf{C}_{A_{T}} \mathbf{x}_{A},
\end{array}
$$

being the matrices $\mathbf{A}_{A_{T}}=\operatorname{diag}\left(\mathbf{A}_{A}, \cdots, \mathbf{A}_{A}\right), \mathbf{B}_{A_{T}}=\operatorname{diag}\left(\mathbf{B}_{A}, \cdots, \mathbf{B}_{A}\right)$ and $\mathbf{C}_{A_{T}}=\operatorname{diag}\left(\mathbf{C}_{A}, \cdots, \mathbf{C}_{A}\right)$ block diagonal, where $\mathbf{A}_{A}, \mathbf{B}_{A}$ and $\mathbf{C}_{A}$ are defined as follows [16]:

$$
\mathbf{A}_{A}=\left[\begin{array}{ccc}
0 & 0 & \epsilon \omega_{A} \\
1 & 0 & \omega_{A}^{2}+2 \zeta_{A} \omega_{A} \epsilon \\
0 & 1 & \epsilon+2 \zeta_{A} \omega_{A}
\end{array}\right], \mathbf{B}_{A}=\left[\begin{array}{c}
0 \\
0 \\
g_{A}
\end{array}\right], \mathbf{C}_{A}=\left[\begin{array}{lll}
0 & 0 & 1
\end{array}\right]
$$

where the actuator is defined by $g_{A}>0$, its damping ratio $\zeta_{A}$ and natural frequency $\omega_{A}$. The value of $\epsilon$ models the low-pass properties of the actuator. The actuator in this work is an APS Dynamics Model 400 electrodynamic shaker, which is shown in Fig. 2(b). The identified parameters of Eq. (9) are [16]: $\omega_{A}=13.2 \mathrm{rad} / \mathrm{s}(2.1 \mathrm{~Hz}), \zeta_{A}=0.5, g_{A}=12000$ and $\epsilon=47.1$. 


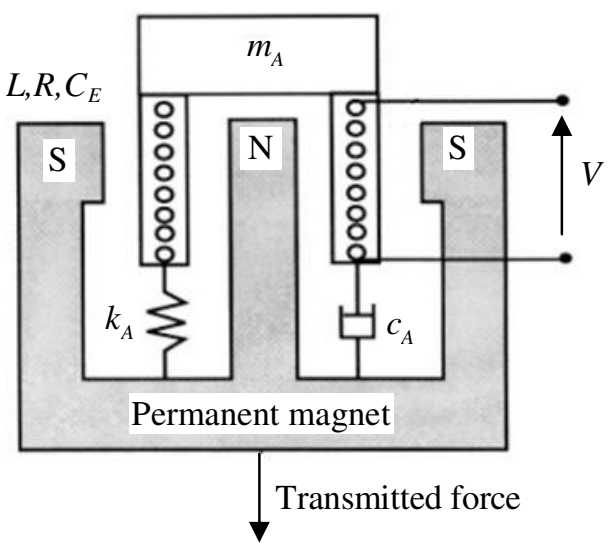

(a) Sketch of typical electrodynamic iner-

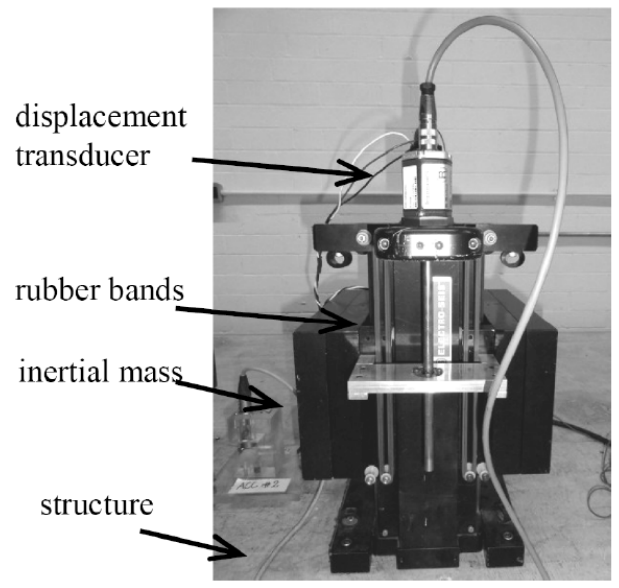

(b) APS Dynamics Model 400 Shaker. tial actuator.

Figure 2: Inertial-mass actuator.

\subsection{State-space model of the closed-loop system}

The state equation of the closed-loop system is obtained from Fig. 1 and Eqs. (1)-(9), and results in

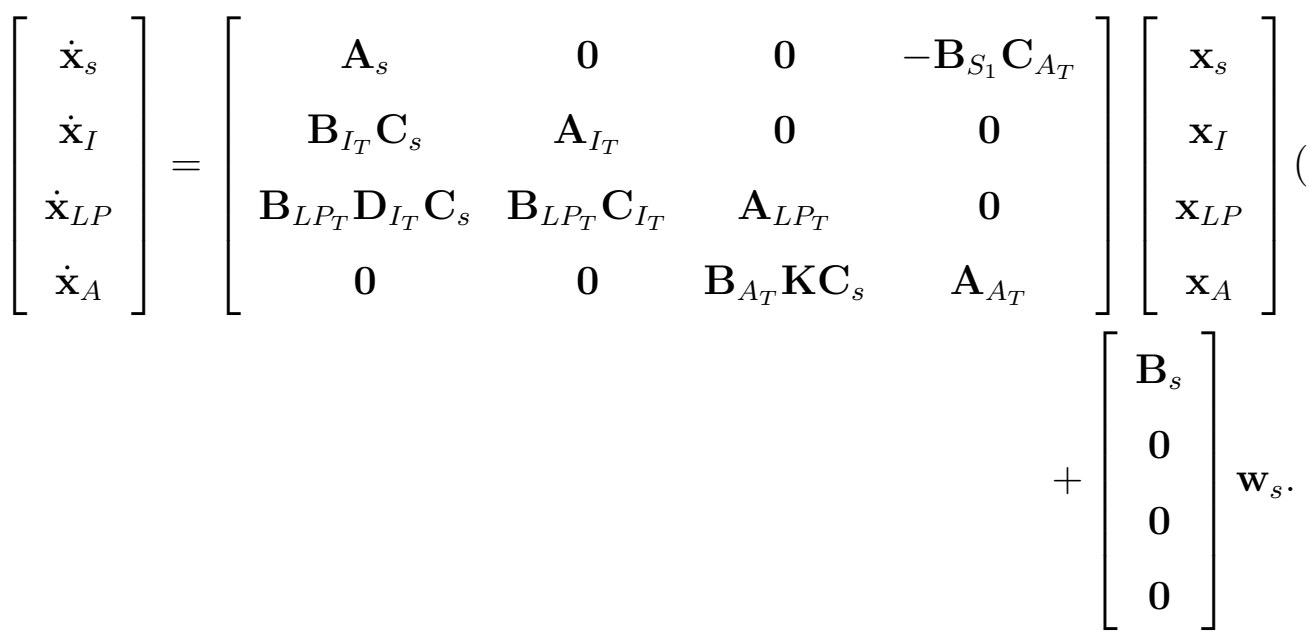

The eigenvalues of the $2(n+2 q+p) \times 2(n+2 q+p)$ state-space matrix are considered into the restrictions defined in the design. These eigenvalues (i.e., the 
poles of the closed-loop system) are denoted by $\zeta_{C L_{\tau}} \omega_{C L_{\tau}} \pm j \omega_{C L_{\tau}} \sqrt{1-\zeta_{C L_{\tau}}^{2}}$, where $\tau \in[1, \cdots, 2(n+2 q+p)]$ and $j$ is the imaginary unit.

\subsection{Human vibration perception}

The vibration that can be perceived by a human depends on the direction of incidence to the human body, the frequency content of the vibration (for a given amplitude) and the duration of sustained vibration, among other factors. The frequency sensitivity variation for a body position can be taken into account by attenuating or enhancing the system response for frequencies where perception is less or more sensitive, respectively. The degree to which the response is attenuated or enhanced is referred to as frequency weighting. Thus, frequency weighting functions are applied in order to account for the different acceptability of vibrations for different directions and body positions. ISO 2631 [11] and BS 6841 [17] provide details for frequency and direction weighting functions that can be applied which are all based on the basicentric coordinate system shown in Fig. 3. These have been included in current floor design guidelines such as the SCI guidance [18]. According to ISO 2631, for z-axis vibration and standing and seating, the frequency weighting function $\left(W_{k}\right)$ is a filter with the frequency response shown in Fig. 4 .

Human comfort under vibration is also related to the duration of sustained vibration [19]. Thus, persistent vibrations should be penalised in the control design, giving more importance to transient vibration of long-duration than those of short-duration. This is taken into account by multiplying the system response by an exponential time weighting (i.e., $e^{\alpha t}$ ), where $\alpha>0$ adds a constraint in the relative stability of the controlled system. Note that 


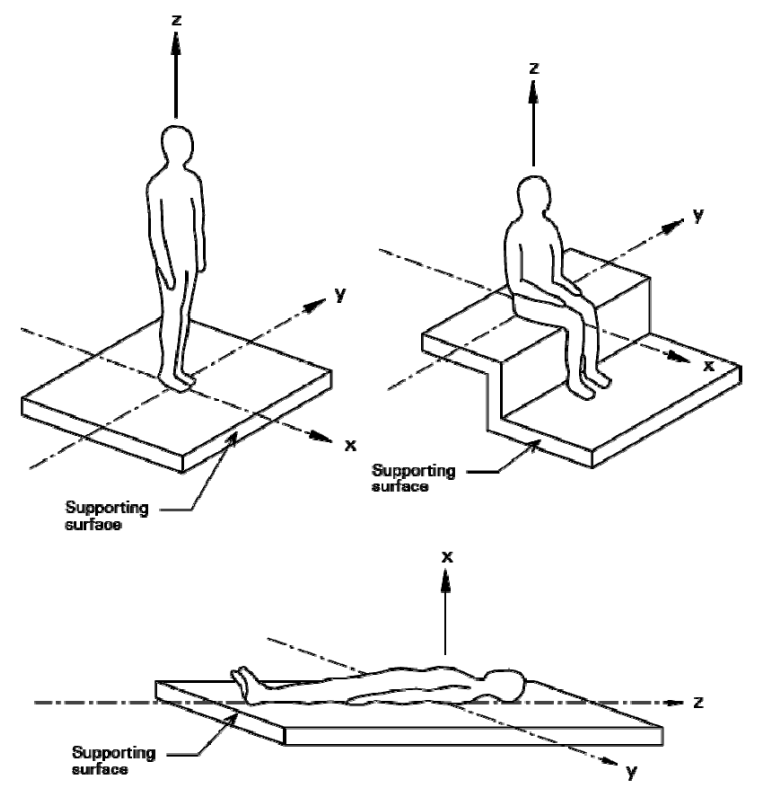

Figure 3: Directions for vibration according to ISO 2631 [11] and BS 6841 [17] (after [18]).

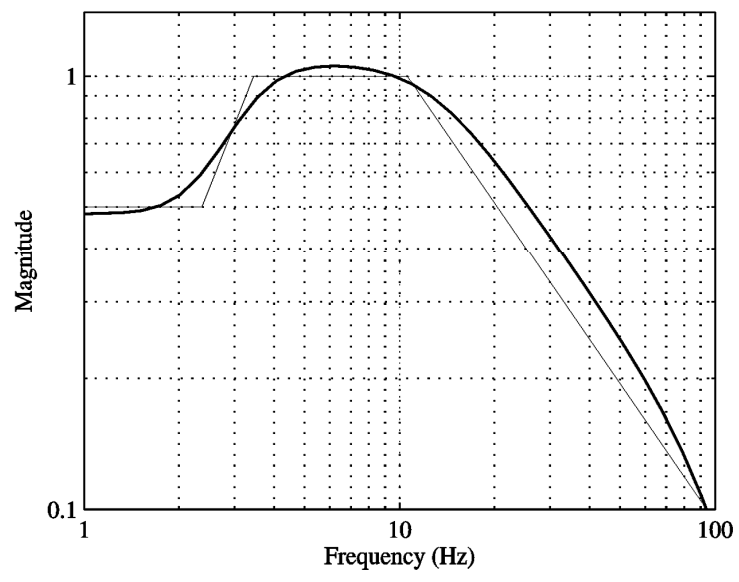

Figure 4: Frequency weighting function $W_{k}$ (thicker curve) and its asymptotic definition (thinner curve) [11]. 
persistent states are penalised more heavily as $\alpha$ is increased.

The human vibration perception is considered in the controller design by weighting the state vector of the structure $\mathbf{x}_{s}=\left[x_{s_{1}}, \cdots, x_{s_{2 n}}\right]$ (see Eq. (1)) as follows:

$$
x_{s_{W_{l}}}=\left(e^{\alpha t} x_{s_{l}}(t)\right) * g_{F W}(t), l \in[1, \cdots, 2 n],
$$

where $(*)$ denotes the convolution process and $g_{F W}(t)$ is the impulse response function of a system with the frequency response function (FRF) shown in Fig. 4. Note that the weighted vector $\mathbf{x}_{s_{W}}$ is only used to calculate the PI used to derive the optimal sensor/actuator locations and the gain matrix. In other words, the weighting functions are not included in the closed-loop system of Fig. 1.

\section{Controller design methodology}

The above section describes the closed-loop system at Eq. (10), where the structure states variables $\mathbf{x}_{s}$ are weighted at Eq. (11). Thus, the vector $\mathbf{x}_{s_{W}}$ depends on the imparted damping by the designed control, the parameter $\alpha$ (i.e., the duration of sustained vibration) and the frequency content of the vibration. Therefore, the control design methodology considers $\mathbf{x}_{s_{W}}$, mode shapes and natural frequencies to define a PI, whose minimum provides the optimal A/S location and gain matrix (Eq. (5)) that maximize vibration reduction regarding the practical issues. This PI is defined as follows:

$$
J(\mathbf{K}, \boldsymbol{\Lambda})=\frac{1}{2} \int_{0}^{t_{f}} \mathbf{x}_{s_{W}}^{T}(\mathbf{K}, \boldsymbol{\Lambda}) \mathbf{Q} \mathbf{x}_{s_{W}}(\mathbf{K}, \boldsymbol{\Lambda}) d t
$$


where the matrix $\mathbf{Q}$ is a $2 n \times 2 n$ positive definite matrix, which is taken as $[9]$

$$
\mathbf{Q}=\left[\begin{array}{cccccc}
\omega_{1}^{2} \phi_{1, \max }^{2} & \cdots & 0 & 0 & \cdots & 0 \\
\vdots & \ddots & \vdots & \vdots & \ddots & \vdots \\
0 & \cdots & \omega_{n}^{2} \phi_{n, \max }^{2} & 0 & \cdots & 0 \\
0 & \cdots & 0 & \phi_{1, \max }^{2} & \cdots & 0 \\
\vdots & \ddots & \vdots & \vdots & \ddots & \vdots \\
0 & \cdots & 0 & 0 & \cdots & \phi_{n, \max }^{2}
\end{array}\right]
$$

in which $\phi_{k, \max }$ is the maximum value of the $k^{\text {th }}$ eigenvector $\phi_{k}$. Note that the displacement states are weighted by the natural frequencies, thus making the displacement states comparable to the velocity states. The variable $\boldsymbol{\Lambda}$ contains the locations of a set of $p$ actuators and $q$ sensors. Finally, the value of $t_{f}$ is the simulation time to obtain the PI, which must be large enough to achieve the steady state of $J(\mathbf{K}, \boldsymbol{\Lambda})$ (i.e., the weighted vector $\mathbf{x}_{s_{W}} \cong 0$ ).

The design methodology consists of defining a set of input arguments to find a set of outputs that minimizes the PI. The input arguments can be group into:

1. System parameters. These parameters are: the models of the flexible structure and inertial actuators, human vibration perception ( $\alpha$ and $\left.g_{F W}(t)\right)$, the system perturbance to assess the controller performance and the set of structural nodes (i.e., possible locations for A/S), which is denoted by $\boldsymbol{\Lambda}_{P I}$.

2. Design parameters. These parameters may be not considered in the optimization because they mainly depend on the open-loop response of the structure and inertial actuators, reducing thus the computational 
cost. These parameters are the low-pass $\left(\omega_{L P}\right)$ and high-pass $\left(\omega_{I}\right)$ filters, the actuator saturation voltage and the minimum value of the damping corresponding to the lower closed-loop poles of the actuator dynamics, which is denoted by $\zeta_{\text {stroke }}$.

The proposed design methodology is divided into the following steps:

Step 1: Obtain the models of the flexible structure and inertial actuators. It can be obtained from a theoretical analysis, a finite element model or an experimental identification.

Step 2: Define parameters of human vibration perception $\left(\alpha\right.$ and $\left.g_{F W}(t)\right)$ where $0 \leq \alpha \leq \min _{k}\left(\zeta_{k} \omega_{k}\right), \forall k \in[1, \cdots, n]$. Note that the upper limit of $\alpha\left(\min _{k}\left(\zeta_{k} \omega_{k}\right)\right)$ guarantees that the system simulation converges to zero. The function $g_{F W}(t)$ is defined in [11].

Step 3: Select the possible values for $\boldsymbol{\Lambda}\left(\boldsymbol{\Lambda}_{P I}\right)$. Note that $\boldsymbol{\Lambda}_{P I}$ can be simplified by a previous analysis, reducing the computational cost. This is useful for complex structures with a large number of nodes.

Step 4: Define the values of $\omega_{L P}, \omega_{I}$ the actuator saturation voltage and $\zeta_{\text {stroke }}$.

Step 5: Define the system perturbance to assess the controller performance. Note that the design of optimal controllers for unknown disturbances is not trivial since prescribed disturbances are needed within the design process. The solution adopted in this work, similar to that used in [9], is to approximate the influence of zero initial conditions and a spatially distributed, but temporally impulsive, disturbance force by 
an appropriate initial condition and zero disturbance force. This is achieved by introducing a non-zero initial condition to the velocity states of the structure. Thus, the system perturbance is defined as $\mathbf{x}_{s}(0)=\left[x_{s_{1}}=0, \cdots, x_{s_{n}}=0, x_{s_{n+1}}=\dot{x}_{s_{1}}(0), \cdots, x_{s_{2 n}}=\dot{x}_{s_{n}}(0)\right]$, where each value of $\dot{x}_{s_{k}}(0)$ is obtained as follows:

$$
\dot{x}_{s_{k}}(0)=F_{0} \phi_{k, \max }
$$

where $F_{0}$ represents the impulse loading applied to a particular vibration mode. Note that the impulsive force is applied to the point of maximum amplitude of each vibration mode, creating thus an extreme scenario for the initial disturbance. It is expected that the control system will perform successfully under other loading conditions.

Step 6: Find the values of $\boldsymbol{\Lambda}$ and $\mathbf{K}$ that minimize $J(\mathbf{K}, \boldsymbol{\Lambda})$ of Eq. (12). Operationally, the Step 4 is divided into the following substeps:

Step 6.1: The values of $J$ are obtained for each $\Lambda \in \Lambda_{P I}$ as follows

$$
J_{\Lambda}=\min _{\mathbf{K}} J(\mathbf{K}, \mathbf{\Lambda}),
$$

where each $J_{\Lambda}$ is calculated by using the MATLAB function fminsearch, which minimises the function defined by the simulation of the control scheme of Fig. 1 with the initial conditions defined by Eq. (14), subjected to the stability of Eq. (10) and the restrictions associated with $\alpha$ and $\zeta_{\text {stroke }}$.

Step 6.2: The final values of $\mathbf{K}$ and $\boldsymbol{\Lambda}$ are those corresponding to the minimum value of $J_{\Lambda}$, which is denoted as $J_{O P}$ and is defined as follows:

$$
J_{O P}=\min _{\Lambda} J_{\Lambda}
$$


It should be remarked that Step 6 can be repeated for different values of parameters defined in Step 4 in order to see the influence of them on $J_{O P}$ and actuator stroke.

\section{Experimental implementation}

This section describes the experimental implementation of the AVC design methodology presented in Section 3 on an in-service indoor walkway. Frequency and time response tests are carried out to examine the control system performance.

\subsection{Structure description}

The test structure, which is a walkway sited in the University of Exeter Forum Building (Fig. 5), is approximately $15 \mathrm{~m}$ long and $2.7 \mathrm{~m}$ wide, as shown in Fig. 6. It was decided that a test grid of 39 test points (TP) would be used (13 x 3 rows) to carry out an experimental modal analysis (EMA). The excitation was provided by an APS Dynamics model 113 shaker, which was placed sequentially at TPs 4 and 7 . The acceleration response was monitored by 13 QA-750 accelerometers. Thus, a total of six measurement setups were taken for the modal test: three sets with roving accelerometers for two actuator positions.

Data acquisition was carried out using a Data Physics Mobilyzer II digital spectrum analyser controlled using the SignalCalc software. Its configuration was as follows: i) the actuator was driven with a random signal with frequency band $0-80 \mathrm{~Hz}$, ii) the response was monitored with frequency span 0-80 Hz and iii) a 10 second block size was used (hence frequency resolution $0.1 \mathrm{~Hz}$ ) with $75 \%$ overlap using a Hanning window and stable averaging to 
acquire data for approximately 4 minutes, which was the length of time required to achieve sufficiently clean FRFs upon averaging out the effects of unmeasured extraneous excitation.

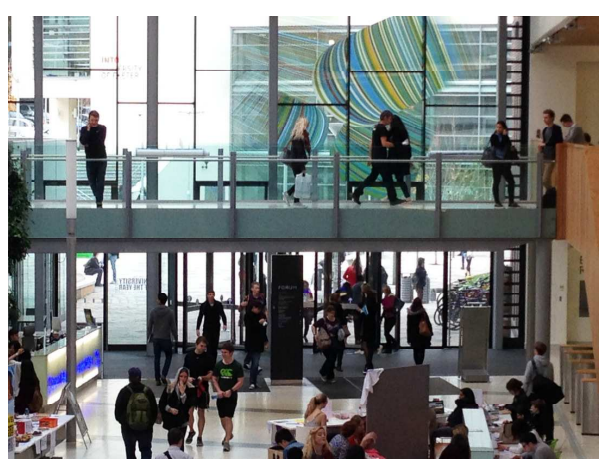

(a) General view.

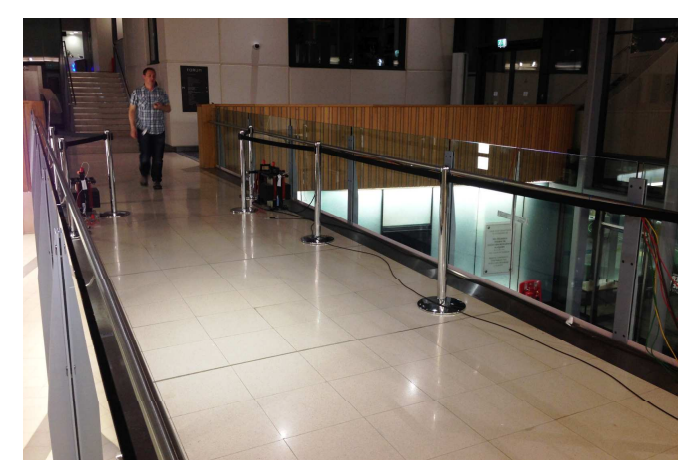

(b) Walking area.

Figure 5: Forum walkway (University of Exeter).

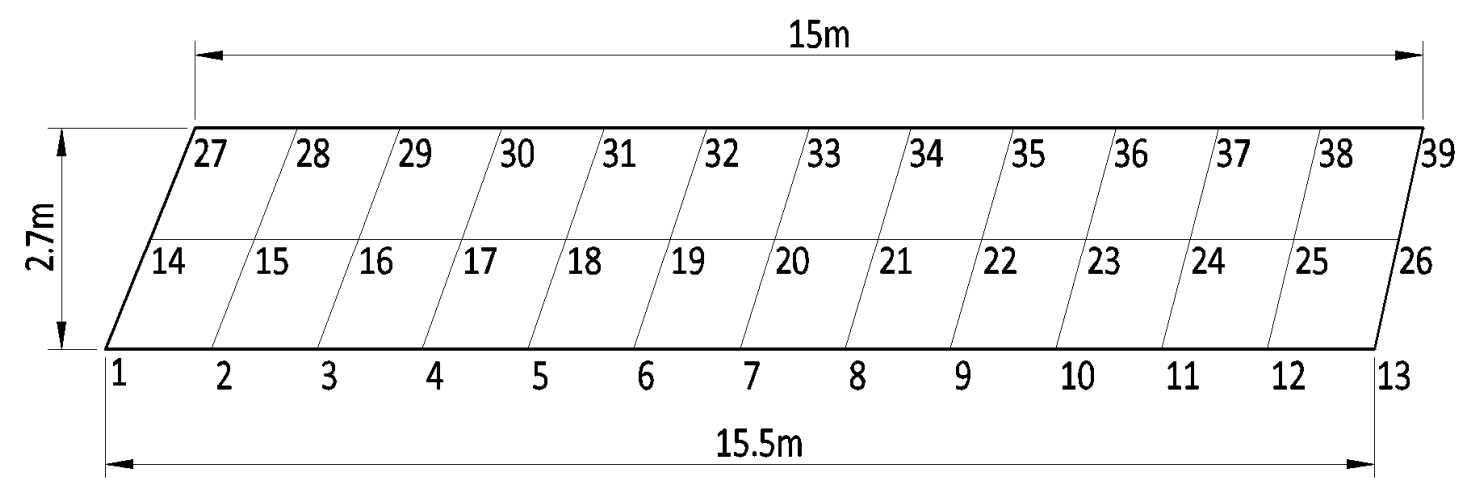

Figure 6: Test grid for the EMA.

The FRF data obtained were analysed using the ME'scope modal parameter estimation software to determine the structural modal properties from EMA. Here, the multiple reference polynomial method was used for curve 
fitting the FRF data. These results were also verified by performing an analysis with the multiple reference AF polynomial method (a method based on the stability of system poles). The properties of the first four modes obtained are presented in Table 1 and the mode shapes are shown in Fig. 7. The good accuracy of the model can be observed in Fig 8, which shows a comparison between the experimental point accelerance FRF at TP 7 and its regenerated counterpart based on the estimated modal properties.

Table 1: Modal properties for the first four vibration modes of the walkway.

\begin{tabular}{|l|cccc|}
\hline $\begin{array}{l}\text { Mode Number } \\
\text { (bending or }\end{array}$ & $\begin{array}{c}\text { Natural } \\
\text { Frequency } \\
\text { torsional) }\end{array}$ & $\begin{array}{c}\text { Damping } \\
\text { Ratio }\end{array}$ & $\begin{array}{c}\text { Modal } \\
\text { Mass }\end{array}$ & $\phi_{k, \max }$ \\
\hline 1 (bending) & 6.3 & 1.0 & 15 & $66.5 \cdot 10^{-6}$ \\
2 (torsional) & 10.5 & 0.9 & 10 & $98.5 \cdot 10^{-6}$ \\
3 (bending) & 14.6 & 2.1 & 39 & $25.6 \cdot 10^{-6}$ \\
4 (bending) & 20.5 & 2.5 & 12 & $84.8 \cdot 10^{-6}$ \\
\hline
\end{tabular}




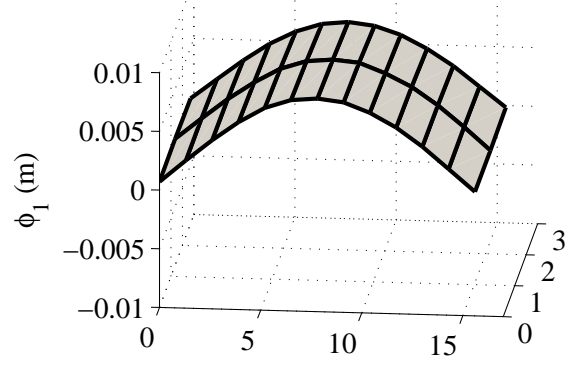

(a) First vibration mode (bending).

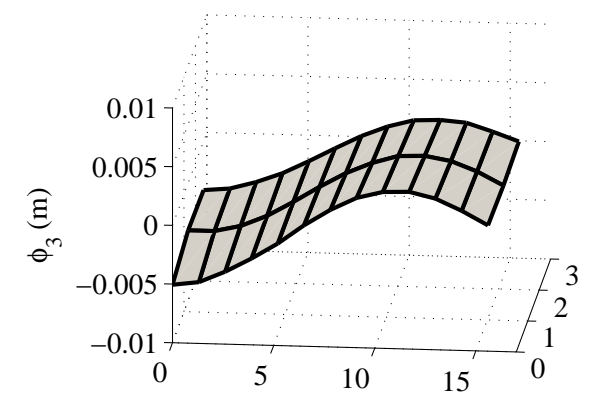

(c) Third Vibration mode (bending).

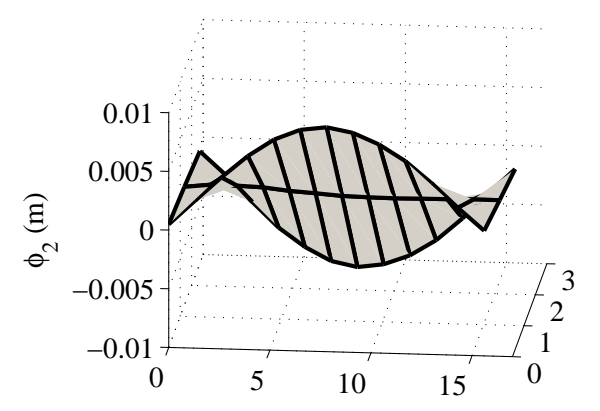

(b) Second Vibration mode (torsional).

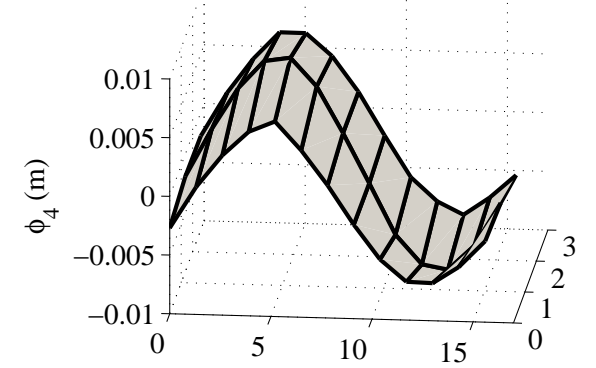

(d) Fourth Vibration mode (bending).

Figure 7: Mode shapes of the first four vibration modes. 


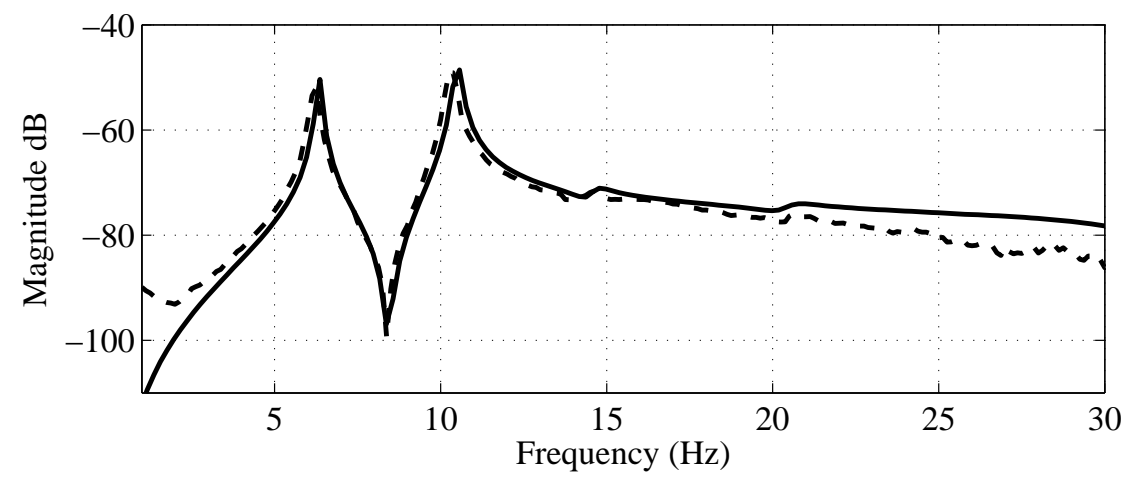

(a) Magnitude in $\mathrm{dB}$ referenced to $\left(\mathrm{m} / \mathrm{s}^{2}\right) / N$.

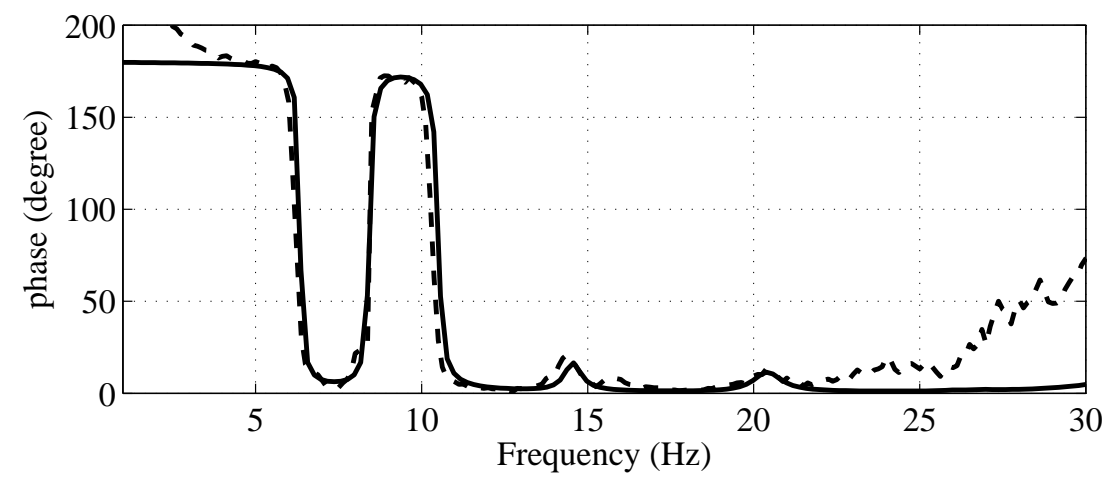

(b) Phase.

Figure 8: FRF of the Forum walkway at TP 7. (- -) Experimental results and (-) model. 


\section{2. $A V C$ design}

The objective of this section is to demonstrate the viability of the design methodology by designing and implementing a SISO DVF and a MIMO DVF with two sensors and actuators for the structure described above. The stepby-step controller design methodology is followed from now on.

Step 1: The structure and the inertial actuators models are defined into Sections 4.1 and 2.1, respectively.

Step 2: The value of $\alpha$ is chosen as 0.25 .

Step 3: The structural nodes $\left(\boldsymbol{\Lambda}_{P I}\right)$ considered in the design Step 1 are the 39 TPs used in the EMA (see the test grid of Fig. 6).

Step 4: The configuration is $\zeta_{\text {stroke }}=0.35, \omega_{I}=2 \pi \cdot 2 \mathrm{rad} / \mathrm{s}$ and $\omega_{L P}=$ $2 \pi \cdot 30 \mathrm{rad} / \mathrm{s}$. The actuator saturation voltage is chosen as $2 \mathrm{~V}$, which is maximum allowable saturation voltage, and $1.5 \mathrm{~V}$. It can be noted that the values of actuator saturation voltage are chosen as long as the risks of stroke saturation and instability due to spillover are avoided by choosing properly $\omega_{I}$ and $\omega_{L P}$, respectively. On the one hand, the value of $\omega_{I}$ depends on the existing tradeoff between the sensitivity at low frequencies (i.e. risk of stroke saturation) and the influence of the low-frequency dynamics on DVF control performance. On the other hand, the value of $\omega_{L P}$ must guarantee the finite gain property of the control loop at high frequencies, avoiding spillover problems [10], whereas the significant vibration modes, which are the two first ones, can be damped by the used DVF. Thus, the values of $\omega_{I}=2 \pi \cdot 2 \mathrm{rad} / \mathrm{s}$ 
and $\omega_{L P}=2 \pi \cdot 30 \mathrm{rad} / \mathrm{s}$ are obtained by a preliminary design, where the stability, the damping performance and the risk of stroke saturation are studied.

Step 5: Three different values for the impulse force $F_{0}$ are used: 100, 1000 or $2670 \mathrm{~N}$. Note that the value of $2670 \mathrm{~N}$ is the normalised maximum force of an idealised heel-drop excitation [19].

Step 6: The stability condition of the closed-loop system of Eq. (10) is used to define the initial conditions for $\boldsymbol{K}$ that minimises each $J_{\Lambda}$.

Tables 2 and 3 show the values of $J_{O P}$ and the maximum stroke for the three values of $F_{0}, \omega_{I}=2 \pi \cdot 2 \mathrm{rad} / \mathrm{s}, \omega_{L P}=2 \pi \cdot 30 \mathrm{rad} / \mathrm{s}$. In addition, these tables consider two values of actuator saturation voltage $(1.5$ and $2 \mathrm{~V})$ in order to show its influence on the controller performance and the maximum actuator stroke. Moreover, the structure is modelled by the first seven vibration modes. The following conclusions can be derived from these tables: i) a MIMO AVC reduces the energy of the system more than a SISO AVC for the same design conditions ( $F_{0}$ and actuator saturation voltage), ii) the stroke saturation is reduced if the actuator saturation voltage is equal to $1.5 \mathrm{~V}$ but the energy of the system $\left(J_{O P}\right)$ is increased (i.e., the damping performance is worse), iii) the stroke saturation is not a problem in this practical implementation since the maximum of these designs $(52 \mathrm{~mm})$ is less than the maximum actuator stroke (65 mm) and iv) the gain matrix $\boldsymbol{K}$ can be chosen as diagonal because $K_{12}=K_{21}$ are negligible compared with $K_{11}$ and $K_{22}$.

The AVC designs chosen to be implemented were SISO with gain $K_{11}=$ 926 placed at node 7 and MIMO with gains $K_{11}=625$ and $K_{22}=769$ placed 
Table 2: Optimal SISO designs for various initial forces and saturation voltages.

\begin{tabular}{|c|c|c|c|c|c|}
\hline $\begin{array}{c}F_{0} \\
(\mathrm{~N})\end{array}$ & $\begin{array}{c}\text { Sat } \\
(\mathrm{V})\end{array}$ & $\mathrm{A} / \mathrm{S}$ node & $K_{11}$ & $J_{O P}$ & $\begin{array}{c}\text { Stroke } \\
(\mathrm{mm})\end{array}$ \\
\hline 100 & 2 & 34 & 1042 & $1.97 \cdot 10^{-5}$ & 31 \\
100 & 1.5 & 34 & 1042 & $2.36 \cdot 10^{-5}$ & 21 \\
1000 & 2 & 33 & 973 & $7.11 \cdot 10^{-3}$ & 28 \\
1000 & 1.5 & 33 & 973 & $7.92 \cdot 10^{-3}$ & 20 \\
2670 & 2 & 7 & 926 & $6.84 \cdot 10^{-2}$ & 52 \\
2670 & 1.5 & 7 & 926 & $7.23 \cdot 10^{-2}$ & 39 \\
\hline
\end{tabular}

Table 3: Optimal MIMO designs for various initial forces and saturation voltages.

\begin{tabular}{|c|c|c|c|c|c|}
\hline $\begin{array}{c}F_{0} \\
(\mathrm{~N})\end{array}$ & $\begin{array}{c}\text { Sat } \\
(\mathrm{V})\end{array}$ & A/S nodes & $K_{11}, K_{22}, K_{12}$ & $J_{O P}$ & $\begin{array}{c}\text { Stroke } \\
(\mathrm{mm})\end{array}$ \\
\hline 100 & 2 & $(32,33)$ & $(555,448,-2)$ & $1.39 \cdot 10^{-5}$ & 18 \\
100 & 1.5 & $(32,33)$ & $(506,492,-2)$ & $1.61 \cdot 10^{-5}$ & 18 \\
1000 & 2 & $(33,34)$ & $(830,155,-5)$ & $5.22 \cdot 10^{-3}$ & 40 \\
1000 & 1.5 & $(33,34)$ & $(830,155,-5)$ & $6.00 \cdot 10^{-3}$ & 29 \\
2670 & 2 & $(7,33)$ & $(625,769,-3)$ & $5.64 \cdot 10^{-2}$ & 51 \\
2670 & 1.5 & $(7,33)$ & $(631,764,-3)$ & $6.17 \cdot 10^{-2}$ & 39 \\
\hline
\end{tabular}

at nodes 7 and 33. The design corresponding to $F_{0}=2670 \mathrm{~N}$ was chosen since it is representative of a worst-case scenario of loading. If the control system is safe (regarding stroke saturation) for this case, it will be safe under a variety of loading scenarios. Paying attention to both tables, it can be observed that a saturation voltage of $2 \mathrm{~V}$ improves the PI $(5 \%$ for SISO 
and $9 \%$ for MIMO). Besides, it can be observed that the MIMO designs always improve upon the SISO designs. For instance, for the design chosen, the PI for the MIMO design is $21 \%$ smaller than the one of SISO. Finally, the simulated FRFs of the Forum walkway at TP 7 are shown in Fig. 9, where the imparted damping of both controllers can be seen. These show that MIMO AVC is better than SISO AVC in terms of magnitude around the first and second vibration mode.

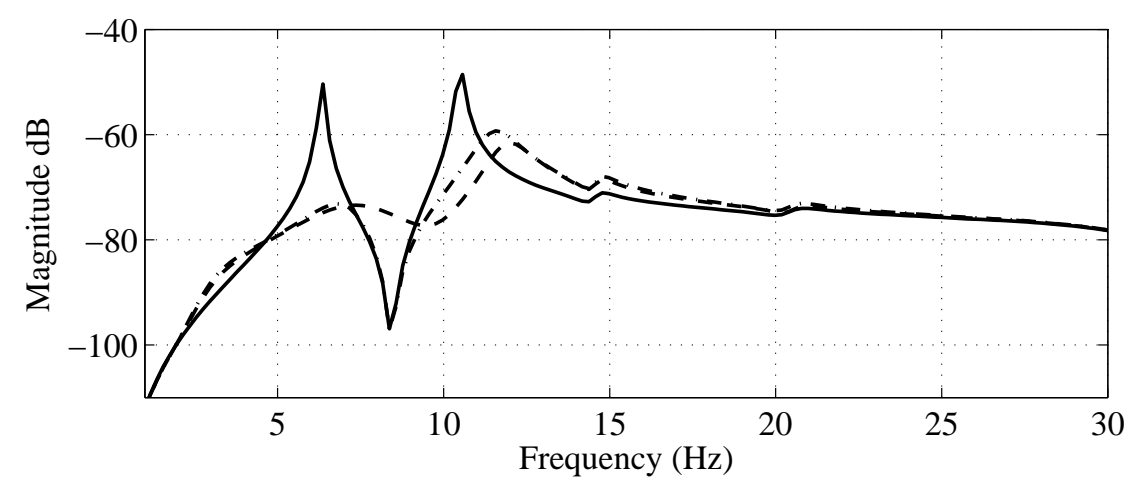

Figure 9: Simulated FRF's of the Forum walkway at TP 7. (-) Without control, (- -) MIMO and (-.-) SISO. Magnitude in dB referenced to $\left(m / s^{2}\right) / N$.

\subsection{Frequency and time response tests}

The objective of this section is to show that both SISO and MIMO AVC can be implemented in practice. The FRF response test was carried out by placing an APS Dynamics model 113 shaker at TP 7. The configuration of the data acquisition was the same as used in subsection 4.1. Fig. 10 shows the experimental FRF of the Forum walkway at TP 7 , where it can be seen that the MIMO AVC performed better than the SISO AVC in terms 
of magnitude around the first and second vibration mode. In addition, if the FRFs obtained at TP 7 are compared against the simulated FRFs of Fig. 9, it can be seen that the simulation and experimental data match quite well.

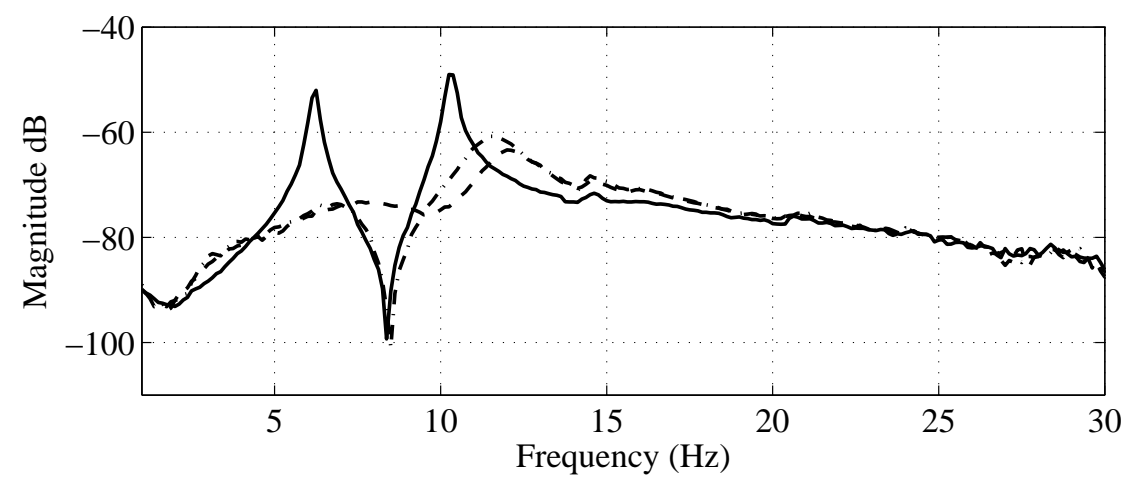

Figure 10: Experimental FRF of the Forum walkway at TP 7. (-) Without control, (- -) MIMO and (-.-) SISO. Magnitude in dB referenced to $\left(\mathrm{m} / \mathrm{s}^{2}\right) / N$.

The structure vibration level was also measured by carrying out a single pedestrian walking test with pacing rate of $2.1 \mathrm{~Hz}$ (126 beats per minute). This rate was chosen so that its third harmonic would be $6.3 \mathrm{~Hz}$ and hence excite the first bending mode. The test consisted of a single pedestrian walking from one end of the walkway to the other end and then back again, with the pacing rate controlled by a handheld audible metronome. The results of this measurement are shown in Fig. 11, where the $W_{k}$ weighted response acceleration and the $1 \mathrm{~s}$ running RMS are shown. When the structure was not controlled, the maximum acceleration value was $0.17 \mathrm{~m} / \mathrm{s}^{2}$, the peak $1 \mathrm{~s}$ RMS value was $0.096 \mathrm{~m} / \mathrm{s}^{2}$ and the R-factor was 19.12. This R-factor is obtained by dividing the maximum value of the frequency weighted $1 \mathrm{~s}$ RMS trend of acceleration by $0.005 \mathrm{~m} / \mathrm{s}^{2}$ [17]. When the system was controlled by the MIMO AVC, the maximum acceleration value was $0.04 \mathrm{~m} / \mathrm{s}^{2}$, the peak 
$1 \mathrm{~s}$ RMS value was $0.015 \mathrm{~m} / \mathrm{s}^{2}$ and the R-factor was 3.05. Finally, when the SISO AVC was used, the R-factor increased to 3.66, which was an increase of $20 \%$ compared with the value of R-factor obtained with the MIMO AVC. It must be noted that R-factors of 4 should not be exceeded for a low probability of adverse comments [17]. Therefore, R-factors for single person walking (both MIMO and SISO) can be considered acceptable by the design limits.

The performance of both controllers was also tested using a heel drop excitation, which is an impulsive excitation useful in evaluation transient response of floor structures and also in checking stability properties. These results from these measurements are shown in Fig. 12, where it can be seen that both controllers imparted significant damping compared with the uncontrolled case.

Finally, a group random walking was also measured without control and with the MIMO AVC. Fig. 13 shows 200 s of this test, where the vibration reduction in terms of the maximum acceleration, $\mathrm{R}$-factor and vibration dose value (VDV) can be seen. Also, Fig. 14 shows the corresponding power spectral density (PSD) of response of these group random walking tests. It can be seen that: i) the maximum R-factor is reduced from 14.681 to 4.867 , ii) the VDV is reduced from 0.173 to $0.056 \mathrm{~m} / \mathrm{s}^{1.75}$ and iii) the vibration response between 0 and $20 \mathrm{~Hz}$ was practically eliminated. Finally, an Rfactor equal to 4.867 could be considered acceptable by the design limits because some guidance recommends higher values of 8 , which perhaps in this situation given the bridge/floor nature of the walkway would actually be more appropriate [17]. 


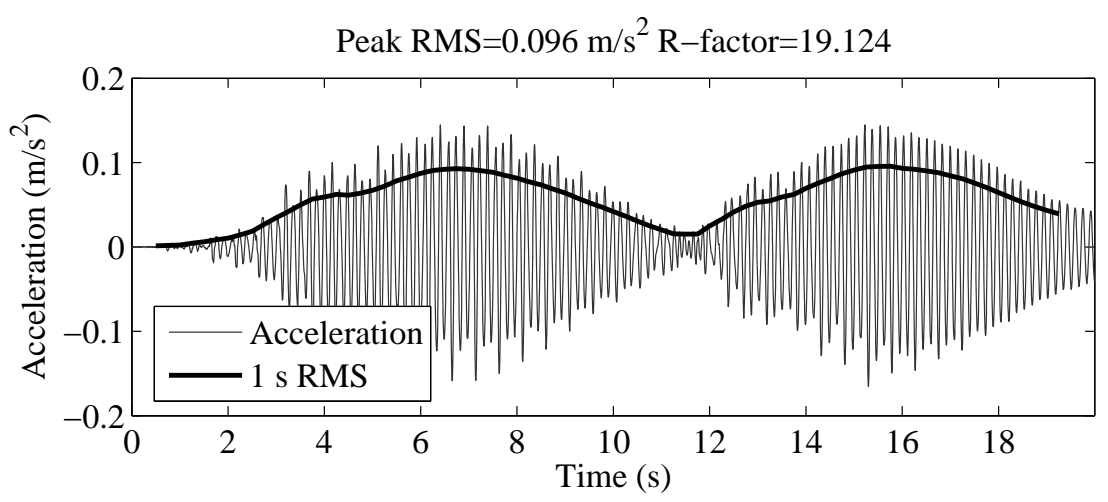

(a) Uncontrolled.

Peak RMS $=0.015 \mathrm{~m} / \mathrm{s}^{2} \mathrm{R}-$ factor $=3.053$

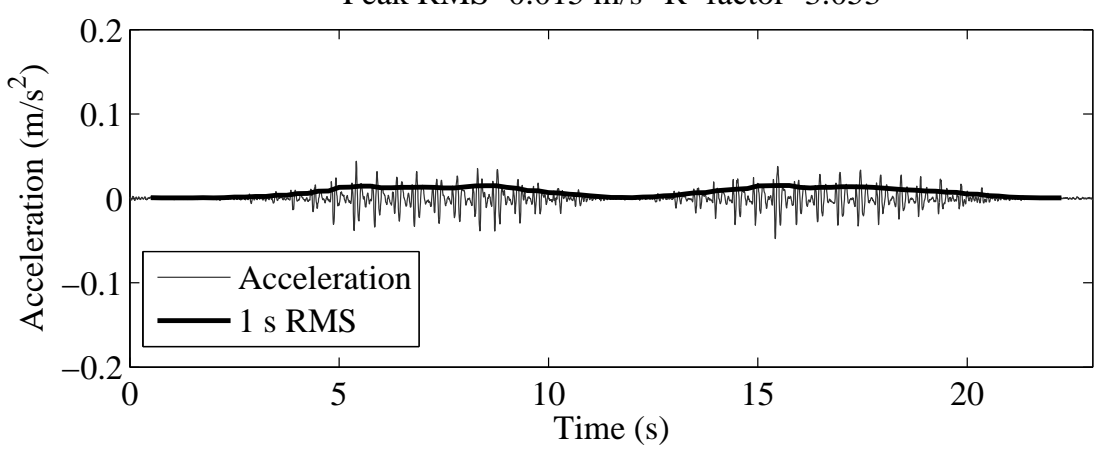

(b) Controlled MIMO.

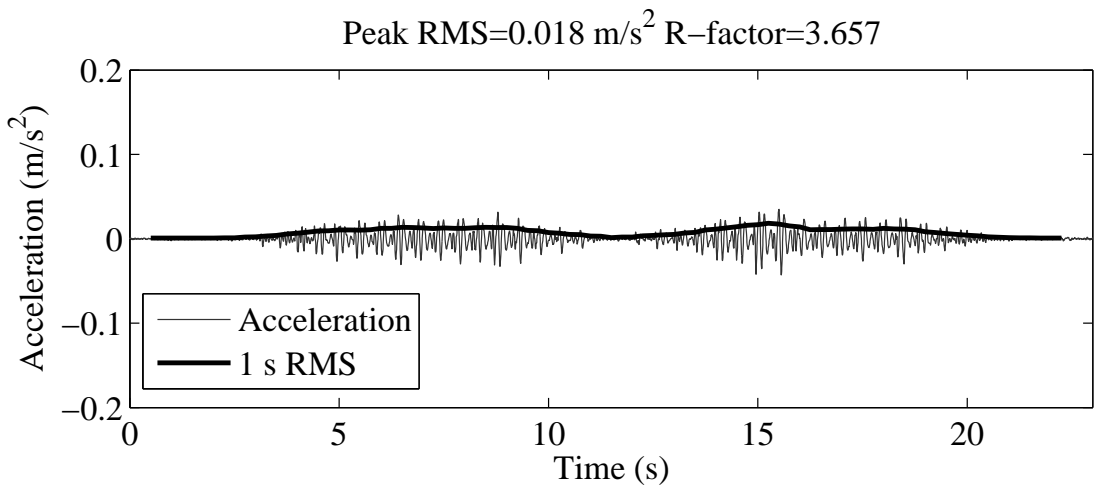

(c) Controlled SISO.

Figure 11: Experimental results. Walking at $2.1 \mathrm{~Hz}$ (126 bpm). 


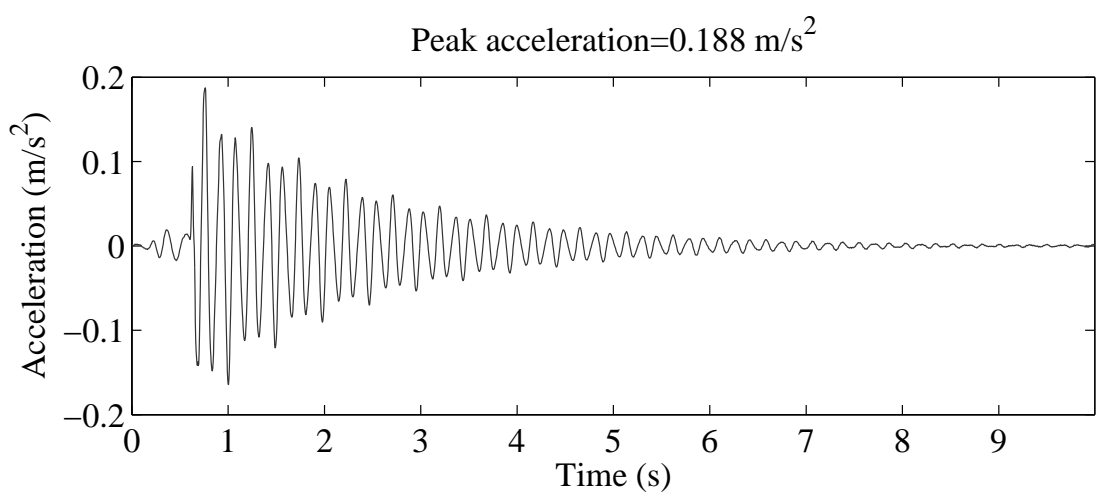

(a) Uncontrolled.

Peak acceleration $=0.126 \mathrm{~m} / \mathrm{s}^{2}$

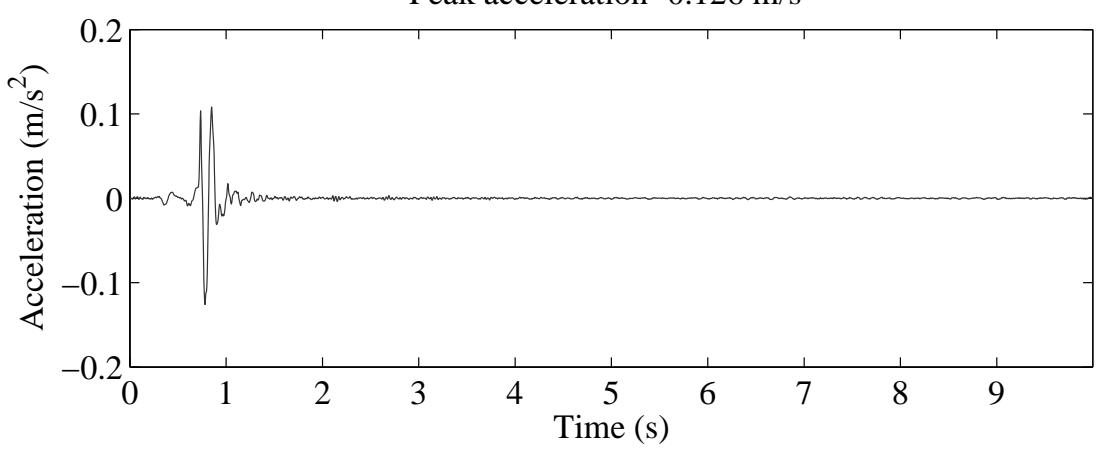

(b) Controlled MIMO.

Peak acceleration $=0.130 \mathrm{~m} / \mathrm{s}^{2}$

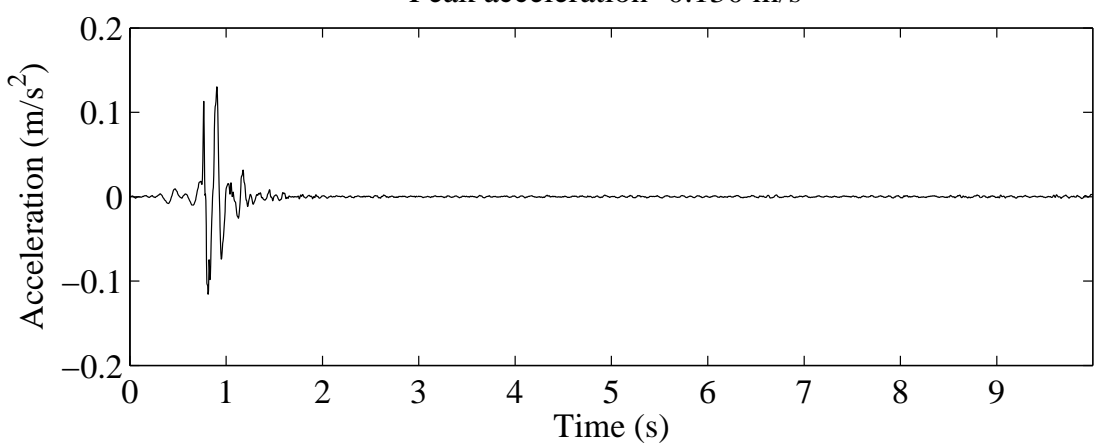

(c) Controlled SISO.

Figure 12: Experimental results. Heel drop. 


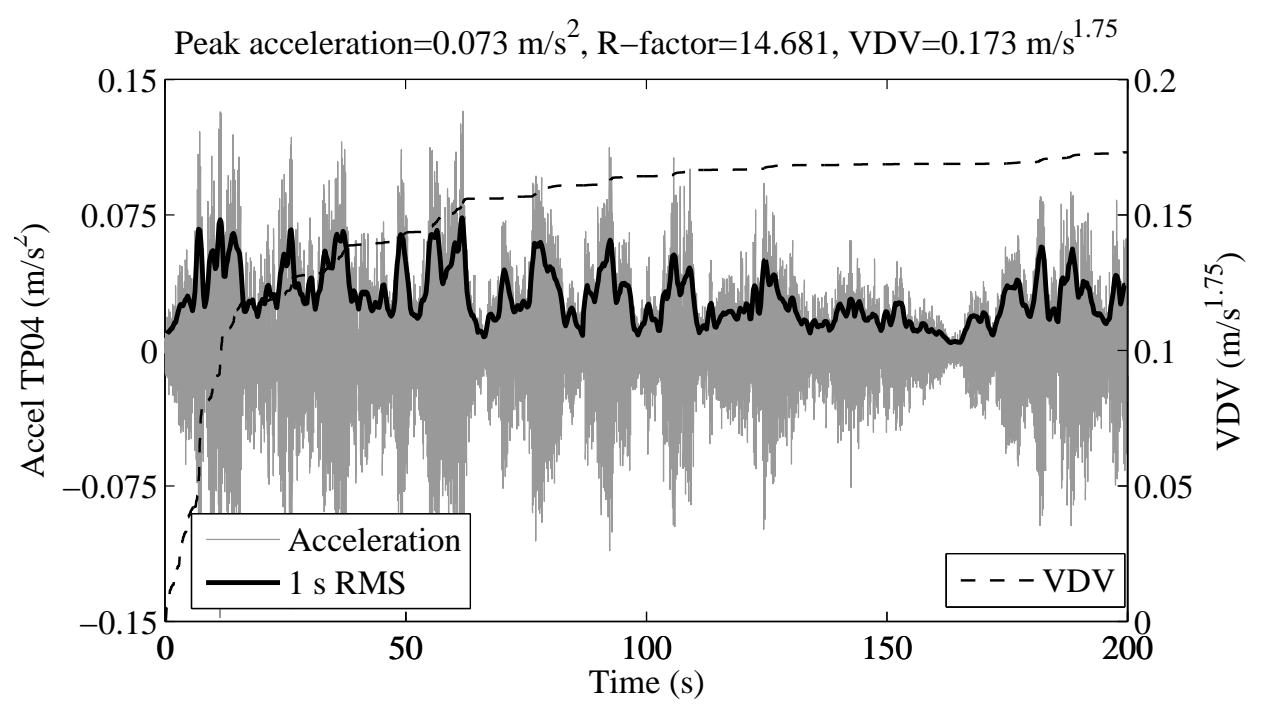

(a) Uncontrolled.

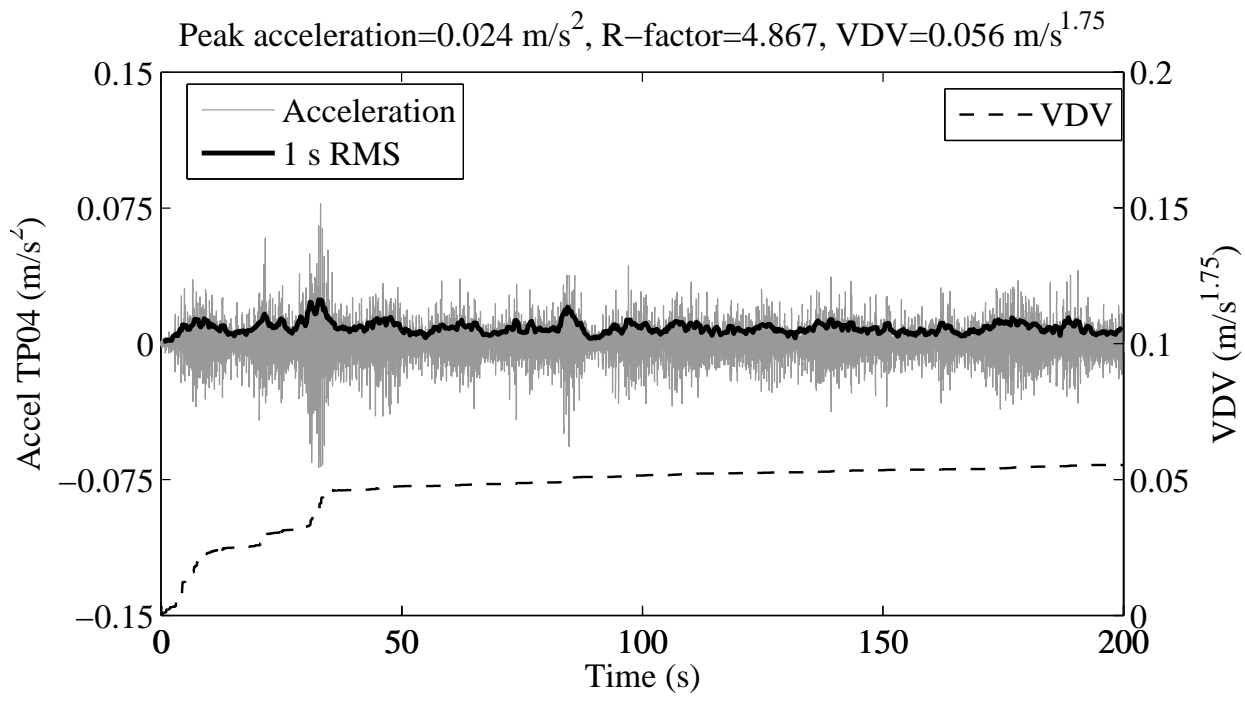

(b) Controlled MIMO.

Figure 13: Experimental results. Group random walking. 


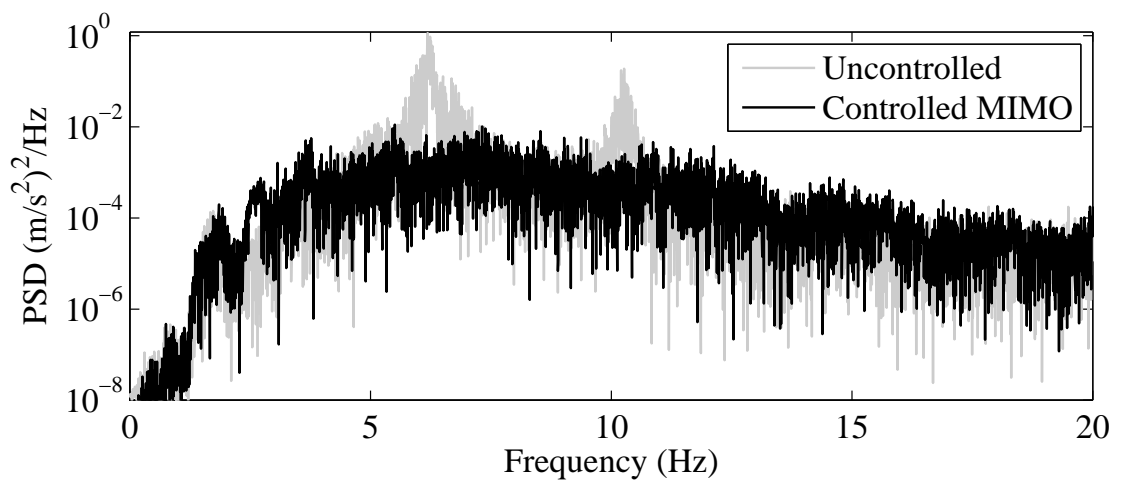

Figure 14: Experimental results. PSD of response of the group random walking. 


\section{Conclusions}

This work has proposed an AVC design methodology for controlling humaninduced vibration based on DVF and an optimal control. That is, a PI is minimised in order to design simultaneously the controller parameters and the actuator/sensor location. The methodology is totally general since it can be considered within a SISO or MIMO strategy. Furthermore, it is implementable in the sense that all the significant practical issues are carefully considered within the design process. That is, actuator dynamics, actuator saturations, spillover effects and human perception dependency to frequency and excitation time have been taken into account.

Results from experimental measurements conducted on an in-service indoor walking have illustrated the viability of the presented methodology with both SISO and MIMO design. It was observed that MIMO control improved the results compared with SISO, even though the dynamics of this structure are not representative of a typical floor structure with multiple vibration modes locally spatially distributed, which is where MIMO strategies find their particular interest. Hence, with the experience from this relatively simple experimental application, the authors plan to apply this methodology to other structures with more complicated dynamics, particularly multi-bay floor structures with high modal density.

\section{Acknowledgment}

The authors acknowledge the financial support provided by the Fundación Caja Madrid through the grant "II Convocatoria de Becas de Movilidad para profesores de las universidades públicas de Madrid durante el curso 
académico 2012/2013" and also the UK Engineering and Physical Sciences Research Council (EPSRC) though grant EP/J004081/2 entitled "Advanced Technologies for Mitigation of Human-Induced Vibration".

The authors would also like to thank Mr Wai Kei Ao, Mr Vasileios Karakonstantis, Miss Jie Niu and Dr Donald Nyawako for their help in the experimental work.

\section{References}

[1] J. Brownjohn, C. Middleton, Procedures for vibration serviceability assessment of high-frequency floors, Engineering Structures 30 (6) (2008) $1548-1559$.

[2] H. Bachmann, Lively footbridges - a real challenge., in: Proceedings of the International Conference on the Design and Dynamic Behaviour of Footbridges, 2002.

[3] A. Ebrahimpour, R. L. Sack, A review of vibration serviceability criteria for floor structures, Computer and Structures 83 (28-30) (2005) 24882494.

[4] M. J. Hudson, P. Reynolds, Implementation considerations for active vibration control in the design of floor structures, Engineering Structures 44 (2012) 334-358.

[5] C. Moutinho, A. Cunha, E. Caetano, Analysis and control of vibrations in a stress-ribbon footbridge, Structural Control and Health Monitoring 18 (6) (2011) 619-634. 
[6] I. M. Díaz, E. Pereira, P. Reynolds, Integral resonant control scheme for cancelling human-induced vibrations in light-weight pedestrian structures, Structural Control and Health Monitoring 19 (2012) 55-69.

[7] I. M. Díaz, E. Pereira, M. J. Hudson, P. Reynolds, Enhancing active vibration control of pedestrian structures using inertial actuators with local feedback control, Engineering Structures 41 (1) (2012) 157-166.

[8] M. J. Hudson, P. Reynolds, D. Nyawako, Efficient design of floor structures using active vibration control, in: ASCE Structures Congress, Vol. 401, 2011.

[9] L. M. Hanagan, E. C. Kulasekere, K. S. Walgama, K. Premaratne, Optimal placement of actuators and sensors for floor vibration control, Journal of Structural Engineering 126 (12) (2000) 1380-1387.

[10] W. M. Griggs, D. O. B. Andersona, A. Lanzon, A "mixed" small gain and passivity theorem in the frequency domain, Systems and Control Letters 56 (9-10) (2007) 596-602.

[11] ISO2631-1, Mechanical vibration and shock - Evaluation of human exposure to whole-body vibration. Part 1: General requirements, International Organization for Standardization, 1997.

[12] S. S. Aphale, A. J. Fleming, S. O. R. Moheimani, Integral resonant control of collocated smart structures, Smart Materials and Structures 16 (2) (2007) 439-446.

[13] W. K. Gawronski, Advanced Structural Dynamics and Active Control of Structures, Springer-Verlag, 2004. 
[14] J. Middlehurst, Practical Filter Design, Prentice Hall, 1993.

[15] A. Preumont, Vibration control of active structures: an introduction, Kluwer Academic Publishers, 2002.

[16] I. M. Díaz, P. Reynolds, Acceleration feedback control of human-induced floor vibrations, Engineering Structures 32 (1) (2010) 163-173.

[17] BS6841, Guide to measurement and evaluation of human exposure to whole-body mechanical vibration and repeated shock, British Standards Institute, 1997.

[18] A. L. Smith, S. J. Hicks, P. J. Devine, Design of floors for vibration: A new approach ( P354), The Steel Construction Institute, 2007.

[19] K. Lenzen, Vibration of steel joist-concrete slab floors, Engineering Journal, AISC 3 (3) (1966) 133-136. 